\title{
Identification of $H G D$ mutations in an alkaptonuria patient: using the Internet to seek rare diseases
}

\author{
Sang-Yeun Cho and Ja Hye Kim* \\ Department of Pediatrics, Hanyang University College of Medicine, Seoul, Korea
}

\begin{abstract}
Alkaptonuria (AKU, OMIM: 203500) is a rare autosomal recessive disorder of tyrosine metabolism due to a defect of enzyme activity, homogentisate 1,2-dioxygenase (HGD). The patients with AKU initially presented with dark urine discoloration, and ochronosis and arthritis develop after third decades of life. With advances of Internet resources, web-based health seekers for rare disease are increasing. Here, we report the case of an 18-year-old boy with AKU who visited our center due to dark black urine based on self-diagnosis via web searching of this rare condition. Compound heterozygous mutations in HGD gene, IVS5+3A $>$ C and IVS12+6T $>C$ were identified and both of mutations were detected in his parents. Our case illustrates the utility of publicly available Internet resources for diagnosis of rare disease.
\end{abstract}

Key words: Alkaptonuria, Homogentisate 1,2-dioxygenase, Ochronosis, Tyrosine.

\section{Introduction}

Alkaptonuria (AKU, OMIM: 203500) is a rare autosomal recessive disorder of tyrosine metabolism caused by a deficiency of homogentisate 1,2-dioxygenase (HGD) activity. This enzyme is responsible for converting homogentisic acid (HGA) to maleylacetoacetic acid in the tyrosine degradation pathway [1]. The major clinical findings of AKU are the dark urine, ochronosis, and arthritis of the spine and larger joints which is caused by accumulation of HGA and its oxidation products on connective tissue and joints [1]. Urine discoloration is apparent since young childhood, but darkening may not occur for several hours after voiding. Ochronosis and arthritis occur after third decades of life. The effective treatment for AKU is not exist, but administration of 2-nitro-4-trifluoromethylbenzoyl-1,3-cyclohexanedione or nitisinone has been proposed $[2,3]$. With the expansion of the
Internet resources, it has resulted in wide-spread public scientific information, particularly in rare diseases. We present a case of AKU patient described with two novel HGD mutations who visited Hanyang University Medical Center based on self-diagnosis via web searching.

\section{Case}

An 18-year-old boy visited our center for dark grey discoloration of urine. He was born at full term of gestation to non-consanguineous and healthy Korean parents after an uneventful pregnancy and delivery. His chief complaint was dark black urine since infant period. His parents have consulted general pediatricians, but they have diagnosed him as uric aciduria. General pediatricians had only performed a routine urine analysis at infant period. His symptoms have persisted until adolescence, thus the

Received: May 14 2018, Revised: June 7 2018, Accepted: June 8 2018, Published: 30 June 2018

*Corresponding author: Ja Hye Kim, M.D. (iD http://orcid.org/0000-0002-2477-5199

Department of Pediatrics, Hanyang University Medical Center, Hanyang University College of Medicine, 222-1 Wangsimni-ro, Seongdong-gu, Seoul 04763, Korea.

Tel: +82-42-350-4268, Fax: +82-42-350-4240, E-mail: whiyoo@gmail.com

Conflict of interest: The authors declare that they do not have any conflicts of interest.

(c) This is an open-access article distributed under the terms of the Creative Commons Attribution Non-Commercial License (http://creativecommons.org/licenses/by-nc/4.0/) which permits unrestricted non-commercial use, distribution, and reproduction in any medium, provided the original work is properly cited.

(c) Copyright 2018 by the Korean Society of Medical Genetics and Genomics 
patient have search his symptoms on the Internet. He identified his urine color as similar to patients with AKU, and he concluded he might suffer from AKU.

His height was $176.7 \mathrm{~cm}$ (50th to 75th percentile), and weight was $61.7 \mathrm{~kg}$ (50th percentile). On physical examination, ochronosis was found on right external ear concha. However, neither other skin color change nor joint abnormalities was found. There was no cardiac murmur by heart auscultation. Because the patient and we suspected him strongly as AKU, he was performed urine analysis of $H G A$ and $H G D$ gene analysis at the first visit. Random urine level of HGA was above than $500 \mathrm{mmol} / \mathrm{mol}$ creatinine (reference, $<2 \mathrm{mmol} / \mathrm{mol}$ creatinine). Because commercial single gene testing for $H G D$ is not available in our country, we performed targeted exome sequencing for differential diagnosis. DNA sequencing was performed using the Illumina Miseq platform (Illumina, Inc., San Diego, CA, USA) and the TruSight One sequencing panel (IIlumina, Inc.), which includes 125,395 probes targeting a 12-Mb region spanning 4,813 genes. Sequencing reads were aligned to human reference genome (hg19) using Burrow-Wheeler alignment program (BWA, ver. 0.7.12). Genome Analysis Toolkit (GATK, ver. 3.5) and Sequence Alignment/Map tools (SAM, ver. 0.1.19) were used for single nucleotide polymorphism variant calling from aligned sequence reads. After removing duplicates with PICARD (https://broadinstitute.github.io/picard/), annotation was done by Variant Effect Predictor. Compound heterozygous mutations were identified in the HGD gene: IVS5+3A>C; IVS12+6T>C (Fig. 1). The former splicing mutation was previously reported [4] and the latter is novel variant. The patient's unaffected parents were carriers (Fig. 1). Since the parent's siblings did not have any symptoms, they and their parents did not want to carry out urine or genetic testing. The novel splice variant, IVS12+6C>T was not detected in normative population databases (1000 Genomes browser, http://browser.1000genomes.org/; NHLBI Exome Sequencing Project Exome Variant Server (http://evs.gs.washington.edu/
EVS/; gnomAD, http://gnomad.broadinstitute.org/) and was predicted deleterious on acceptor site using In Silico software programs including NNSPLICE 0.9 version (http://www.fruitfly. org/seq_tools/splice.html) and EX-SKIP (http://ex-skip.img.cas. cz/).

After the diagnosis, we described the potential complications and disease course that may occur to the patient. Because he lives in United States, we recommended subsequent follow-up evaluation there.

\section{Discussion}

The HGD gene mutation results in deficient levels of functional HGD, which is leads to excess levels of HGA [5]. Large amounts of HGA lead to dark urine on exposure to oxygen, which is caused by the oxidation and polymerization of HGA [6]. Also, $\mathrm{HGA}$ and its oxidation products accumulate in connective tissue leading to ochronosis [1]. Ochronotic arthritis is a longstanding finding in patients with AKU [1]. Usually, joint symptoms and cardiac involvement are reported in the third and fourth decades of life, therefore joints and cardiac evaluations are recommended. Initially, however, dark urine is first symptom in infant to adolescence period and is known to occur early phase in this disease before a joint or connective tissue manifestations [6]. As our case, dark urine can be the only clinical feature of AKU patients, so clinician needs to perform a differential diagnosis.

The HGD gene consists of 14 exons and encodes 445 amino acids [7]. To date, more than 110 different disease-causing mutations have been reported (http://www.hgmd.cf.ac.uk). Missense substitutions and splice site mutations are common types of mutations. The splicing mutation of IVS $5+3 \mathrm{~A}>\mathrm{C}$ was reported in a Chinese Han child with AKU, which is a donor splice site of the exon 5 and exon-intron junction [4]. No apparent correlation between genotype and the level of excreted HGA was reported [8]. It might be explained that for a patient to manifest AKU

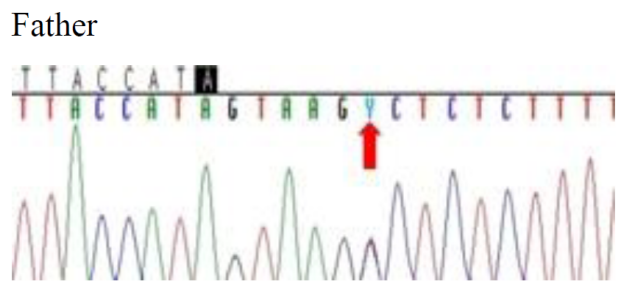

IVS12+6T $>\mathrm{C}$
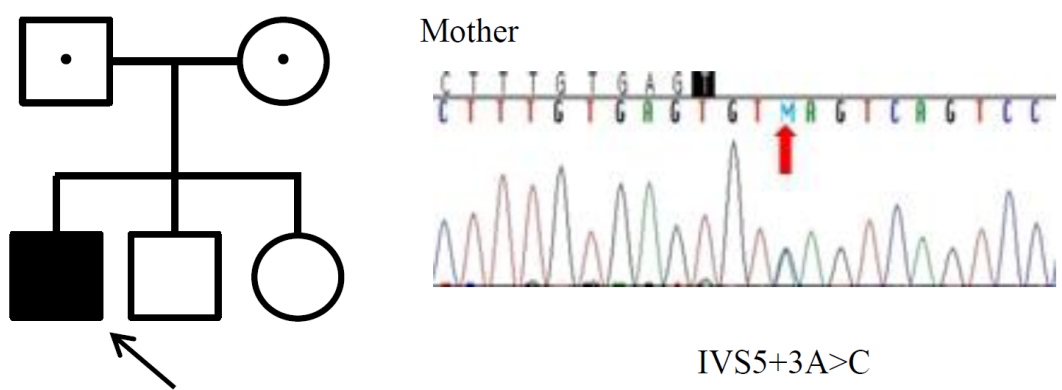

IVS5 $+3 \mathrm{~A}>\mathrm{C}$

Fig. 1. The partial DNA sequence of the homogentisate 1,2-dioxygenase gene and family pedigree. The patient is carrying compound heterozygous mutations for c.IVS5+3A>C and c.IVS12+6T>C. pedigree of the patient. 
symptoms, a loss of more than 99\% of the residual enzymatic activity is required, thus most patients have similar functional activity of HGD [8].

The therapeutic options for patients with AKU remain challenging and limited to palliative analgesia and arthroplasty. Nitisone, a competitive inhibitor of the enzyme 4-hydroxyphenylpyruvate dioxygenase, decreased the formation of HGA. This drug has been used in patients with tyrosinemia type I $[9,10]$. Recent study for nitisone administration of 2 years in patients with AKU decreased urinary HGA excretion [11]. The large scaled and long-term study on clinical effect on cardiac, renal, and joint should be needed of administration of nitisone.

This case demonstrates the utility of publicly available Internet search engines for rare metabolic disease. The open resources on rare diseases would have the potential of increasing the understanding of rare diseases and improving diagnostics. He has observed continuous urine discoloration, thus he found the urine photographs in google to find his disease. Similarly, recent study has been reported that some lysosomal storage disease including Fabry disease and mucopolysaccharidosis type I were diagnosed using Internet-based searching by patients [12]. Because the prevalence of these genetic disease is too low and the many physicians have not experienced of this disease, and rather patients diagnosed by themselves who are highly interested in their health state. These cases showed patients role is changing from passive recipients of medical care to active consumers of health services.

AKU was first reported in 2006 and diagnosed by urine homogentistic acid level measurement without genetic analysis in South Korea [13]. Since then, there has been a report of ochronotic arthropathy in the Department of Orthopedic Surgery, and no genetic analysis has been conducted [14]. As far as we know, this is the first case confirmed by genetic diagnosis.

In conclusion, this case is the first genetically confirmed AKU patient in Korea with a novel variant in HGD gene. This case illustrates the utility of publicly available Internet resources for diagnosis of rare disease.

\section{References}

1. Phornphutkul $C$, Introne WJ, Perry MB, Bernardini I, Murphey MD,
Fitzpatrick DL, et al. Natural history of alkaptonuria. N Engl J Med 2002;347:2111-21.

2. Al Essa M, Al-Shamsan L, Rashed MS, Ozand PT. Alkaptonuria: case report and review of the literature. Ann Saudi Med 1998;18:442-4.

3. Anikster $Y$, Nyhan WL, Gahl WA. NTBC and alkaptonuria. Am J Hum Genet 1998;63:920-1.

4. Li H, Zhang $K, X u$ Q, Ma L, Lv X, Sun R. Two novel mutations in the homogentisate-1,2-dioxygenase gene identified in Chinese Han Child with alkaptonuria. J Pediatr Endocrinol Metab 2015;28:453-6.

5. Braconi D, Millucci L, Bernardini G, Santucci A. Oxidative stress and mechanisms of ochronosis in alkaptonuria. Free Radic Biol Med 2015;88:70-80.

6. Peker $E_{1}$ Yonden Z, Sogut S. From darkening urine to early diagnosis of alkaptonuria. Indian J Dermatol Venereol Leprol 2008;74:700.

7. Fernández-Cañón JM, Peñalva MA. Molecular characterization of a gene encoding a homogentisate dioxygenase from Aspergillus nidulans and identification of its human and plant homologues. J Biol Chem 1995;270:21199-205.

8. Vilboux T, Kayser M, Introne W, Suwannarat P, Bernardini I, Fischer $\mathrm{R}$, et al. Mutation spectrum of homogentisic acid oxidase (HGD) in alkaptonuria. Hum Mutat 2009;30:1611-9.

9. Suwannarat $P, O$ B Brien $K$, Perry MB, Sebring N, Bernardini I, KaiserKupfer $\mathrm{Ml}$, et al. Use of nitisinone in patients with alkaptonuria. Metabolism 2005;54:719-28.

10. Introne WJ, Perry MB, Troendle J, Tsilou E, Kayser MA, Suwannarat $P_{1}$ et al. A 3-year randomized therapeutic trial of nitisinone in alkaptonuria. Mol Genet Metab 2011;103:307-14.

11. Milan AM, Hughes AT, Davison AS, Devine J, Usher J, Curtis $S$, et al. The effect of nitisinone on homogentisic acid and tyrosine: a twoyear survey of patients attending the National Alkaptonuria Centre, Liverpool. Ann Clin Biochem 2017;54:323-30.

12. Bouwman MG, Teunissen $\mathrm{QG}$, Wijburg FA, Linthorst GE. 'Doctor Google' ending the diagnostic odyssey in lysosomal storage disorders: parents using internet search engines as an efficient diagnostic strategy in rare diseases. Arch Dis Child 2010;95:642-4.

13. Nam JH, Lee JH, Park KB, Lee $D H$. A case of alkaptonuria: the first case in Korea. Korean J Pediatr 2006;49:329-31.

14. Yoon JR, Kim Y, Yu J, Yang JH. Ochronotic arthropathy: degenerative and complex tear of black meniscus. J Korean Orthop Assoc 2014:49:389-93 